\title{
Classification of the conditionally observable spectra exhibiting central symmetry
}

\author{
Jun-Hua Chen \\ Department of Physics, FNPE, Czech Technical University, \\ Břehová 7, 11519 Prague, Czech Republic \\ e-mail: cjh611@gmail.com \\ Edita Pelantová \\ Department of Mathematics, FNPE, Czech Technical University, \\ Trojanova 13, 11519 Prague, Czech Republic \\ e-mail: pelantova@km1.fjfi.cvut.cz \\ and \\ Miloslav Znojil \\ Nuclear Physics Institute ASCR, \\ 25068 Řž, Czech Republic \\ e-mail: znojil@ujf.cas.cz
}




\section{Abstract}

The emergence of upside-down symmetry of the bound-state energies $E_{1}=-E_{N}$, $E_{2}=-E_{N-1}, \ldots$ has been observed, in strong-coupling regime, in several pseudoHermitian $N$-state quantum systems. We show that such a symmetry assumption also simplifies a combinatorial classification of these systems since non-equivalent hierarchies of complexifications beyond the strong-coupling boundaries induce nonequivalent pairwise links between the energy levels. 


\section{Introduction}

In 2002, Dunne and Shifman [1] noticed and emphasized that in several quasi-exactly solvable models a number of interesting mathematical as well as physical consequences can be deduced from the emergence of the "central symmetry" duality

$$
E_{n}-c_{(\text {lower })}=c_{(\text {upper })}-E_{N-n}, \quad n=0,1, \ldots
$$

between certain low-lying and highly-excited bound states. In an entirely different context, our recent series of papers [2, 3, 4, 5] revealed that the same type of the central symmetry of the spectrum seems to play a decisive role also during an efficient fine-tuning suppression of instabilities (or, if you wish, of "quantum catastrophes") in certain pseudo-Hermitian phenomenological $N$-site chain models.

In our present short note we intend to broaden the scope of the latter set of references by paying attention, in principle, to all the $N$-dimensional pseudo-Hermitian matrix Hamiltonians. In this sense we are going to complement some considerations presented in the latter reference [5]. In particular, we shall return to the problem of the classification of all the possible "conditionally stable" spectra which exhibit the "stability-friendly" symmetry (1). Indeed, only an incomplete solution of this classification problem has been offered, by one of us, in ref. [5].

We shall start by recalling some of the basic ideas of some previous related papers in section 2. We emphasize there that the collapse of pseudo-Hermitian models can be, generically, mediated by the various alternative mergers and subsequent complexifications of certain pairs of the energy levels. Obviously, a necessity emerges of a correct numbering of the non-equivalent "pairings" and possible complexifications.

Our present main result presented in section 3 offers the answer. Some of its consequences will be summarized in section 4 where we re-emphasize that the introduction of the concept of the conditional observability in [5] opened a rich variety of new scenarios of collapse of phenomenological quantum systems.

\section{Confluences of the levels in $2 J$-state models}

In a typical "catastrophic" scenario as sampled in ref. [5] the growth of a parameter in the Hamiltonian $H$ forces some neighboring energy levels to merge and, subsequently, to complexify. A schematic picture of such a situation is provided here by Figure 1 where six levels of some energy spectrum $\left\{E_{n}\right\}_{n=1,2, \ldots, 6}$ are displayed and where the 


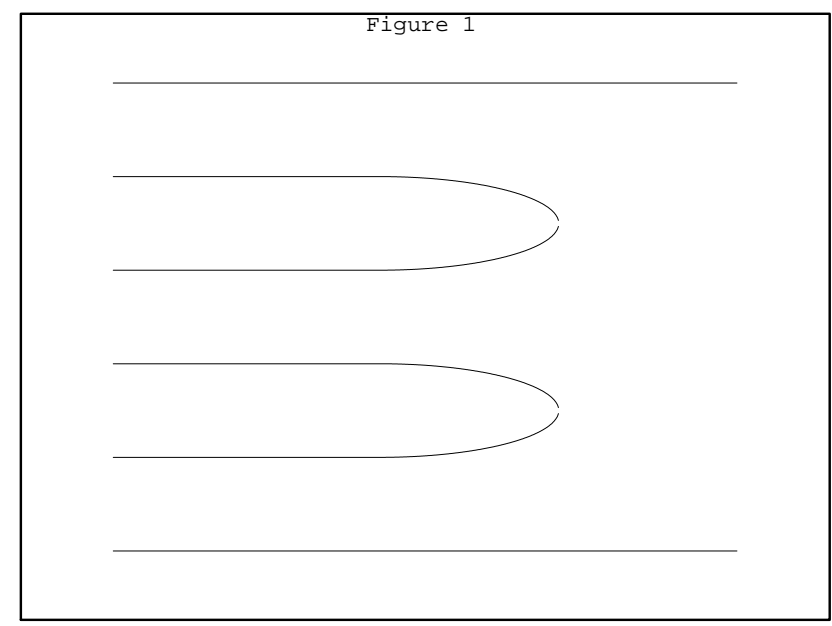

Figure 1: A sample of the mutual attraction of some of the energies followed by their confluence and complexification.

external levels $E_{1}$ and $E_{6}$ are assumed decoupled. We see that when the strength of the non-Hermiticity reaches a critical "exceptional point" value [6], the quantity $E_{2}$ merges with $E_{3}$ while $E_{4}$ merges with $E_{5}$.

Another type of a "catastrophic" scenario can be produced when the attraction between $E_{3}$ and $E_{4}$ dominates. The resulting new forms of the parametric dependence of the spectrum have been sampled in ref. [5] (cf. Figure Nr. 5 there). Here, the message is repeated by Figure 2 which shows that the levels $E_{3}$ and $E_{4}$ merge more quickly than the remaining pair of the levels $E_{2}$ and $E_{5}$.

In Figure 3 we illustrate the most current scenario where the spectrum splits in the separate doublets of levels, each of which reaches its point of confluence separately. Such a scenario has been found to occur in many toy examples. For illustration one could recollect, e.g., the exactly solvable parametric-dependence of the spectrum of the so called $\mathcal{P} \mathcal{T}$-symmetric harmonic oscillator [7] and/or of some of its simplest differential-equation generalizations [8]. In this context, the merging levels are sometimes characterized by the opposite "quasiparities" [7, 9].

We can formulate our first conclusion that while there is just one possible confluence pattern in two-state models [2], the generic four-state models already admit the two distinct complexification patterns as sampled in Figures 1 and 2, Similarly, in all the "next", six-state models one has to contemplate the three substantially different forms of the instability of the system. Their respective complexification patterns can 


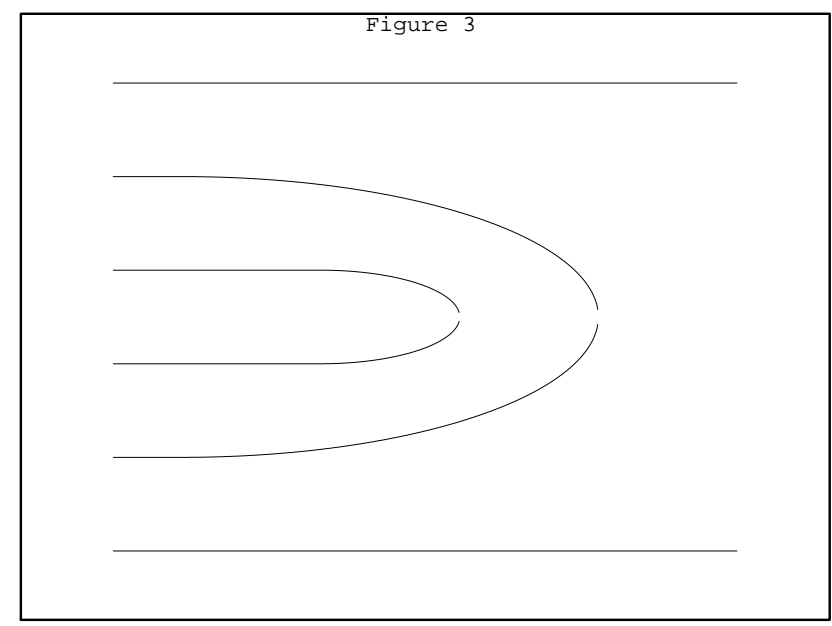

Figure 2: An alternative to Figure 1.

easily be read out of our Figures 1 - 3. In the first two cases it is only necessary to imagine that the effect of the "hidden" attraction only affects the external levels $E_{1}$ and $E_{6}$ somewhere very far to the right, i.e., out of the range of our pictures.

At the higher even dimensions $N=2 J$, the level-linking patterns become perceivably more complicated. Still, up to the dimension as large as $N=2 J=14$, one of us showed in ref. [5] that their classification may still be based on a simplified recurrence relation. In what follows we intend to extend the latter analysis to and beyond the dimension $N=16$. We shall see that the exhaustive and complete enumeration of all the eligible arrangements of the quantum catastrophes remains feasible and that it may be obtained in closed form, provided only that a generating-function approach to the problem is employed.

\section{Counting the non-equivalent scenarios}

\subsection{Notation}

Modifying slightly the notation conventions of ref. [5] let us consider a generic spectrum $E_{1}<E_{2}<\ldots<E_{2 J}$. Let us further assume that sooner or later all these energies complexify, pairwise, with the growth of a suitable parameter $\lambda$. Our task will be an explicit description of the eligible possibilities. Once the symbol $P^{(2 J)}$ denotes the number of the non-equivalent confluence pairings of the levels, we may 


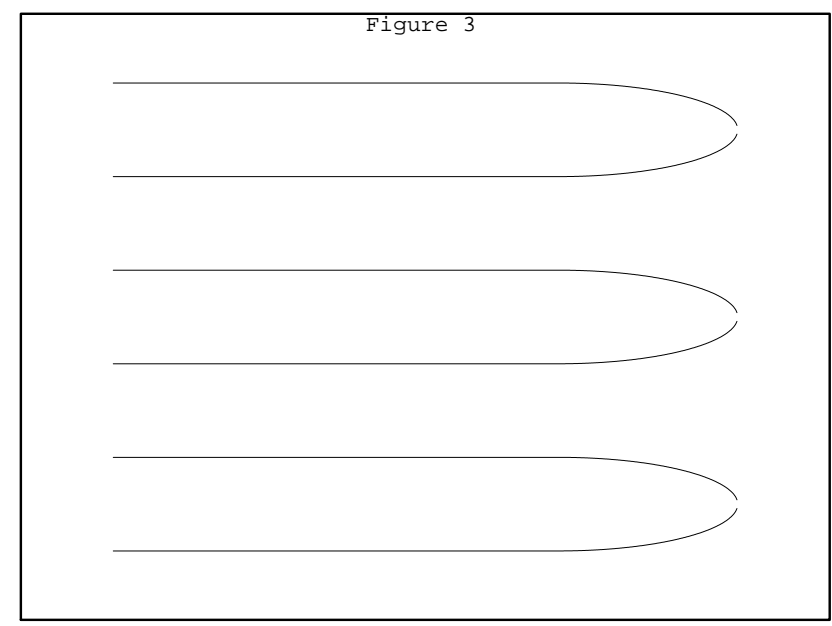

Figure 3: Another alternative to Figure 1.

recollect that $P^{(2)}=1, P^{(4)}=2$ (cf. Figures 1 and 2 once more) and $P^{(6)}=3$.

All the multiple mergers will be ignored as mere trivial limiting cases. Thus, in the slightly modified shorthand notation of ref. [5] the unique merger pattern at $J=1$ will be characterized by the compressed level-number symbol $\{[1,2]\}$. Similarly, at $J=2$ the two symbols $\{[2,3],[4,5]\}$ and $\{[2,5],[3,4]\}$ will be assigned to the two respective possibilities (cf. the inner parts of Figures 1 and 2). Finally, the models with $J=3$ will be assigned the symbols $\{[1,6],[2,3],[4,5]\},\{[1,6],[2,5],[3,4]\}$ and $\{[1,2],[3,4],[5,6]\}$, etc.

\subsection{Classification}

The set of the lower estimates of the quantities $P^{(2 J)}$ as given in Table Nr. 1 of ref. [5] can be replaced by the following complete and exact result.

\section{Theorem.}

At any integer $J=1,2, \ldots$, the number of the non-equivalent patterns of the pairwise

Table 1: Multiplicities $P^{(2 J)}$ of the merging patterns

\begin{tabular}{||c|cccccccccc||}
\hline \hline$J$ & 0 & 1 & 2 & 3 & 4 & 5 & 6 & 7 & 8 & $\ldots$ \\
\hline$P^{(2 J)}$ & 1 & 1 & 2 & 3 & 6 & 10 & 20 & 35 & 70 & $\ldots$ \\
\hline \hline
\end{tabular}


mergers (i.e., confluences and subsequent complexifications) of the energy levels of our $2 J$-state model is given by closed formula,

$$
P^{(2 J)}=\left(\begin{array}{c}
J \\
{\left[\frac{J}{2}\right]}
\end{array}\right) \text {. }
$$

\section{Proof.}

First let us lift the requirement of symmetry, and denote the number of nonequivalent connections as $T^{(2 J)}$. As long as the first energy level can merge with any level $2 i$, their link divides the rest of $2 J-2$ levels into two disconnected groups of $2 i-2$ and $2 J-2 i$ levels. We can write

$$
T^{(2 J)}=\sum_{i=1}^{J} T^{(2 i-2)} T^{(2 J-2 i)}=\sum_{i=0}^{J-1} T^{(2 i)} T^{(2 j-2 i-2)} .
$$

For the generating function

$$
f(x)=\sum_{J=0}^{\infty} T^{(2 J)} x^{J}
$$

we have

$$
f^{2}(x)=\sum_{n=0}^{\infty} \sum_{i=0}^{n} T^{(2 i)} T^{(2 n-2 i)} x^{n}=\sum_{n=0}^{\infty} T^{(2 n+2)} x^{n}=\frac{f(x)-1}{x}
$$

so that we may conclude that

$$
f(x)=\frac{1-\sqrt{1-4 x}}{2 x}, \quad T^{(2 J)}=\frac{(2 J) !}{(J+1) ! J !} .
$$

In the next step let us calculate $P^{(2 J)}$. Firstly, we can write down the recurrences

$$
P^{(2 J)}=P^{(2 J-2)}+\sum_{i=1}^{\left[\frac{J}{2}\right]} T^{(2 i-2)} P^{(2 J-4 i)}=P^{(2 J-2)}+\sum_{i=0}^{\left[\frac{J}{2}\right]-1} T^{(2 i)} P^{(2 J-4 i-4)}
$$

which add the "missing terms" in the simplified lower-estimate relations as employed in ref. [5]. Subsequently, we may employ again the generating function $g(x)$ of $P^{(2 J)}$ and deduce that

$$
g(x)=\sum_{J=0}^{\infty} P^{(2 J)} x^{J}=1+\sum_{J=1}^{\infty} P^{(2 J)} x^{J}
$$

where

$$
\sum_{J=1}^{\infty} P^{(2 J)} x^{J}=\sum_{J=1}^{\infty} P^{(2 J-2)} x^{J}+\sum_{J=2}^{\infty} \sum_{i=0}^{\left[\frac{J}{2}\right]-1} T^{(2 i)} P^{(2 J-4 i-4)} x^{J} .
$$

This enables us to conclude that

$$
g(x)-1=x g(x)+x^{2} f\left(x^{2}\right) g(x) .
$$


After all the insertions we arrive at the final formula

$$
g(x)=\frac{1}{1-x-x^{2} f\left(x^{2}\right)}=\frac{1}{\sqrt{1-4 x^{2}}}+\frac{1-\sqrt{1-4 x^{2}}}{2 x \sqrt{1-4 x^{2}}} .
$$

Our final result $P^{(2 J)}=C_{J}^{\left[\frac{J}{2}\right]}$ (sampled in Table 1) immediately follows. QED.

\section{Summary}

In our present short note we intended to show that and how the purely combinatorial classification of all the possible confluences of the energy levels (i.e., of all the possible quantum catastrophes) can be performed in full generality. We did not work with any particular Hamiltonians this time, noticing only that for constructive purposes, the chain models of refs. [4] exhibiting central symmetry (10) would prove particularly suitable and friendly in technical sense again.

A remark can be added that after the extremely popular choice of the differentialoperator Hamiltonians one often has to refrain just to the most elementary and exceptional "neighboring-level-confluence" scenario [8]. On the basis of our present abstract classification scheme one should expect that the emergence of many less trivial scenarios of the collapse cannot be excluded even in the differential-operator toy models. By sophisticated numerical techniques, some explicit examples have already been discovered recently [10, 11].

\section{Acknowledgement}

Work supported by the MŠMT "Doppler Institute” project Nr. LC06002. 


\section{Table captions}

Table 1. Multiplicities $P^{(2 J)}$ of the merging patterns

\section{Figure captions}

Figure 1. A sample of the mutual attraction of some of the energies followed by their confluence and complexification.

Figure 2. An alternative to Figure 1.

Figure 3. Another alternative to Figure 1. 


\section{References}

[1] G. Dunne and M. Shifman, Annals of Physics 299 (2002) 143.

[2] M. Znojil and H. B. Geyer, Phys. Lett. B 640 (2006) 52;

M. Znojil, Phys. Lett. A 367 (2007) 300.

[3] M. Znojil, Phys. Lett. B 647 (2007) 225.

[4] M. Znojil, J. Phys. A: Math. Theor. 40 (2007) 4863;

M. Znojil, J. Phys. A: Math. Theor., to appear (arXiv: 0709.1569 [math-ph]).

[5] M. Znojil, Phys. Lett. B 650 (2007) 440.

[6] T. Kato, Perturbation Theory for linear Operators (Springer, Berlin, 1966).

[7] M. Znojil, Phys. Lett. A 259 (1999) 220.

[8] D. T. Trinh, J. Phys. A: math. Gen. 38 (2005) 3665.

[9] G. Lévai and M. Znojil, Mod. Phys. Lett. A 16 (2001) 1973;

B. Bagchi, C. Quesne and M. Znojil, Mod. Phys. Lett. A 16 (2001) 2047;

M. Znojil and G. Lévai Mod. Phys. Lett. A 16 (2001) 2273;

A. Sinha and P. Roy, J. Phys. A: Math. Gen. 39 (2006) L377.

[10] P. Dorey, A. Millican-Slater and R. Tateo, J. Phys. A: Math. Gen. 38 (2005) 1305.

[11] Y. Ben-Aryeh and R. Barak, Phys. Lett. A 351 (2006) 388. 